\title{
Two important exceptions to the relationship between energy density and fat content: foods with reduced-fat claims and high-fat vegetable-based dishes
}

\author{
Helen A La Fontaine*, Timothy C Crowe, Boyd A Swinburn and Carl J Gibbons \\ School of Health Sciences, Deakin University, 221 Burwood Highway, Melbourne, Victoria 3125, Australia
}

Submitted 7 July 2003: Accepted 28 0ctober 2003

\begin{abstract}
Objective: To test the hypothesis that many foods with reduced-fat (RF) claims are relatively energy-dense and that high-fat (HF) vegetable-based dishes are relatively energy-dilute.

Design: Nutrient data were collected from available foods in Melbourne supermarkets that had an RF claim and a full-fat (FF) equivalent. Nutrient analyses were also conducted on recipes for HF vegetable-based dishes that had more than 30\% energy from fat but less than 10\% from saturated fat. The dietary intake data (beverages removed) from the 1995 National Nutrition Survey were used for the reference relationships between energy density (ED) and percentage energy as fat and carbohydrate and percentage of water by weight.

Statistics: Linear regression modelled relationships of macronutrients and ED. Paired $t$-tests compared observed and predicted reductions in the ED of RF foods compared with $\mathrm{FF}$ equivalents.

Results: Both FF and RF foods were more energy-dense than the Australian diet and the HF vegetable-based dishes were less energy-dense. The Australian diet showed significant relationships with ED, which were positive for percentage energy as fat and negative for percentage energy as carbohydrate. There were no such relationships for the products with RF claims or for the HF vegetable-based dishes. Conclusion: While, overall, a reduced-fat diet is relatively energy-dilute and is likely to protect against weight gain, there appear to be two important exceptions. A high intake of products with RF claims could lead to a relatively energy-dense diet and thus promote weight gain. Alternatively, a high intake of vegetable-based foods, even with substantial added fat, could reduce ED and protect against weight gain.
\end{abstract}

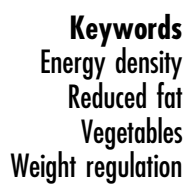

In recent years there has been a dramatic increase in messages about dietary approaches to prevent weight gain or assist with weight loss, particularly about the need to reduce dietary fat intake. These messages have been strengthened by multiple trials that have found that reducing dietary fat intake results in modest weight loss ${ }^{1,2}$.

In response to the rise in public awareness about dietary fat and calls from the nutrition community for more reduced-fat $(\mathrm{RF})$ options $^{3}$, the market has been flooded with products with RF claims ${ }^{4}$. At the same time, messages promoting a high intake of monounsaturated or polyunsaturated fat for the prevention of cardiovascular disease have been constrained for fear of promoting obesity.

In the USA, the parallel trends of increasing availability and consumption of low-fat foods, increasing intake of total energy (but not of dietary fat) and increasing obesity prevalence have been dubbed the 'American paradox ${ }^{3,5,6}$. However, the phenomenon is not restricted to the USA and is also seen in Australia ${ }^{7}$.
Several well-controlled studies have explored the mechanisms by which dietary fat intake might influence body weight ${ }^{8-12}$. A consensus seems to be emerging that the principal mechanism is that diets high in fat are usually energy-dense and that it is the energy density (ED) that promotes 'passive over-consumption' of total energy and thus weight gain $^{13}$. If fat content and ED become dissociated, in other words lose their usual positive relationship, then the usual positive relationship between fat intake and weight gain may also become dissociated.

We hypothesised that two common types of food, products with RF claims and high-fat (HF) vegetable-based dishes, would not exhibit the usual relationships between ED and percentage energy as fat or carbohydrate. We examined the relationships of these foods in the context of the same relationships within the Australian diet. A secondary aim was to examine whether the reduction in fat content in the RF products was associated with a proportional reduction in ED. 


\section{Methods}

All products with a 'Reduced Fat', 'Low Fat' or 'Fat Free' claim, or with product labelling that would lead consumers to believe they were lower in fat (such as 'No Fat', 'Light', 'Lite', 'Skinny' or 'Diet'), were collected from three large supermarkets in high-income areas of Melbourne, Australia. Products were included in the analysis if nutrient data were presented on the packaging and if there was an equivalent full-fat (FF) product available from the same manufacturer. A total of $133 \mathrm{RF} / \mathrm{FF}$ pairs were collected and homogeneous foods were grouped into 63 paired data points for analysis. This involved grouping items with the same product name but produced by different manufacturers (e.g. cheeses and dips) or grouping foods with different flavours but the same manufacturer (e.g. potato chips and yoghurt). The average of the nutritional information was applied to each of these categories. Where products required preparation with additional ingredients, and nutritional information was not available for the 'package only' ingredients, the nutritional data were analysed in the ready to be consumed' state following the manufacturer's directions on the packaging.

The FF/RF food pairs were analysed to determine if the observed decrease in fat content gave a concomitant decrease in ED. The predicted decrease in ED assumed that the grams of fat removed were replaced by equivalent grams of the RF product.

Recipes for HF vegetable-based dishes were obtained from recipe books and internet recipe sites and analysed for nutrient content using Australian food composition databases (AUSNUT) ${ }^{14}$. Dishes were excluded if they contained less than 30\% energy from fat or more than $10 \%$ saturated fat (SFA). This latter criterion was used to ensure that the dishes met current dietary guidelines for SFA ${ }^{15}$. In practice, because vegetable oils contain some SFA and the amount of oil being used was considerable, we needed to exclude recipes with significant meat and dairy products to limit sources of SFA. Examples of dishes included are grilled Mediterranean vegetables with cous cous, cumin rice with eggplant and peppers, layered potato cake, marinated grilled vegetables, and broccoli and tofu stir fry.

The Australian dietary intake data were 24-hour recall data from the 1995 Australian National Nutrition Survey $(\mathrm{NNS} 95)^{7}$ for the population 2 years old and over. All beverages were removed from the data because fluids have very different ED values to solid foods and the physiological recognition of energy intake from fluids appears to be different from that of solids ${ }^{16}$. Fluids that would be consumed as part of foods such as salad dressings, and semi-fluids such as yoghurts, were included.

\section{Statistics}

Data were analysed using the STATA statistical package, version 7 (Stata Corporation, College Station, TX, USA). Linear regression relationships were calculated between ED and percentage energy as fat and carbohydrate and percentage of water by weight for the FF and RF foods and the HF vegetable-based dishes.

A dependent $t$-test was used to compare predicted and observed change in ED from FF to RF products. The predicted decrease in ED assumed that the grams of fat removed from the FF product were replaced by equivalent grams of the lower-fat product.

To provide a population-based dietary context for the various food comparisons, NNS95 data were used to plot linear regression lines which respectively modelled the association of ED with percentage energy as fat, percentage energy as carbohydrate and percentage water by weight. The regression lines were accompanied with 95\% prediction bands (encompassing 95\% of the population data points), and provide a visual reference to the likely range of ED and nutrient intakes in the Australian population's diet. These were plotted and used to represent the Australian population relationships as a dietary context for the relationships within the food products.

The population data were not statistically compared with the food data because they were fundamentally different and there were substantial differences in sample sizes.

\section{Results}

The composition of the FF and RF foods, HF vegetablebased dishes and nutrient intake of the Australian diet are presented in Table 1 . RF foods were significantly lower in fat and ED compared with their equivalent FF products $(P<0.0001)$; however, the average ED of the RF foods was higher than that of the average Australian diet. Carbohydrate content was higher in the RF products than

Table 1 Composition of foods with reduced-fat claims, their full-fat equivalents, high-fat (HF) vegetable-based dishes, and the non-beverage content of the Australian diet (mean \pm standard deviation)

\begin{tabular}{lcccc}
\hline & $\begin{array}{c}\text { Australian } \\
\text { diet }\end{array}$ & $\begin{array}{c}\text { Reduced-fat } \\
\text { foods }\end{array}$ & $\begin{array}{c}\text { Full-fat } \\
\text { foods }\end{array}$ & $\begin{array}{c}\text { HF vegetable-based } \\
\text { dishes }\end{array}$ \\
\hline$n$ & 10794 & 63 & 63 & 47 \\
Energy density $\left(\mathrm{kJ} \mathrm{g}^{-1}\right)$ & $5.1 \pm 1.6$ & $7.7 \pm 5.5$ & $10.2 \pm 6.5$ & $3.9 \pm 1.5$ \\
Energy as fat $(\%)$ & $32.5 \pm 8.9$ & $30.8 \pm 25.6$ & $50.5 \pm 22.6$ & $53.9 \pm 13.9$ \\
Energy as carbohydrate (\%) & $44.8 \pm 10.1$ & $52.3 \pm 25.2$ & $36.8 \pm 22.4$ & $31.3 \pm 12.4$ \\
Weight as water (\%) & $72.3 \pm 7.9$ & $63.1 \pm 26.6$ & $58.2 \pm 25.9$ & $79.4 \pm 8.2$ \\
\hline
\end{tabular}


in the FF foods $(P<0.0001)$ and the population diet. The water content of the RF products was similar to that of the FF products $(P<0.20)$, but these were both substantially lower than in the Australian diet. On the other hand, HF vegetable-based dishes had on average more than $50 \%$ of energy from fat, but were less energy-dense than the Australian diet. These dishes were low in energy from carbohydrate but were on average almost $80 \%$ water by weight.

The decrease in ED was predicted by assuming that the grams of fat removed from the FF product were replaced by equivalent grams of the RF product. On average, the observed reduction in ED from FF to RF food pairs was not significantly different from the predicted values. There was, however, a substantial spread of data points around the line of identity (Fig. 1), indicating a much higher or lower reduction in observed ED for the reduction in fat content.

Figure 2 shows the relationships between ED and percentage energy as fat, percentage energy as carbohydrates and percentage water by weight for the RF foods. The data points for the RF products have been superimposed on the relationships seen in the Australian diet.

For RF products there was no relationship between ED and percentage energy as fat $(\beta=0.04,95 \%$ confidence interval (CI) -0.02 to 0.09 ) (Fig. 2a). In contrast, the population diet showed a significant positive relationship between fat content and ED ( $\beta=0.112$, 95\% CI $0.108-$ 0.116). FF products also demonstrated a significant positive relationship between ED and percentage energy as fat ( $\beta=0.08,95 \%$ CI $0.01-0.15$; data not shown).

There was no relationship between percentage energy as carbohydrate and ED for RF products $(\beta=-0.01,95 \%$



Fig. 1 Predicted compared with observed reduction in energy density from the full-fat foods to their reduced-fat equivalents. The solid line is the regression line and the dashed line is the line of identity (where predicted reduction equals observed reduction). Points below the line of identity indicate that the observed reduction in energy density was less than would be predicted given the reduction in fat content from the full-fat to the reducedfat foods. No statistical difference between predicted and observed
CI -0.06 to 0.05$)$, whereas the population dietary data $(\beta=-0.047,95 \% \mathrm{CI}-0.051$ to -0.043$)$ and the $\mathrm{FF}$ products $(\beta=-0.07,95 \% \mathrm{CI}-0.15$ to 0.00 ; data not shown) showed significant negative relationships (Fig. 2b).

(a)

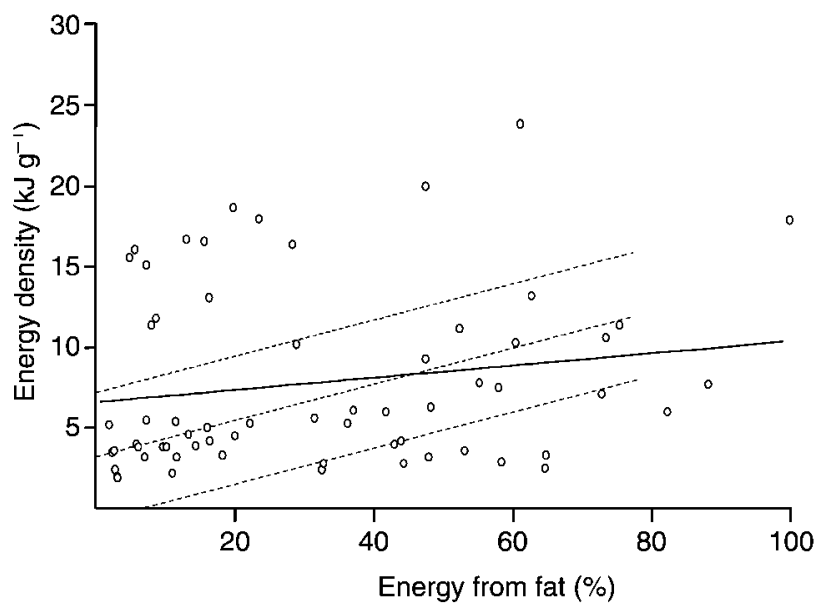

(b)

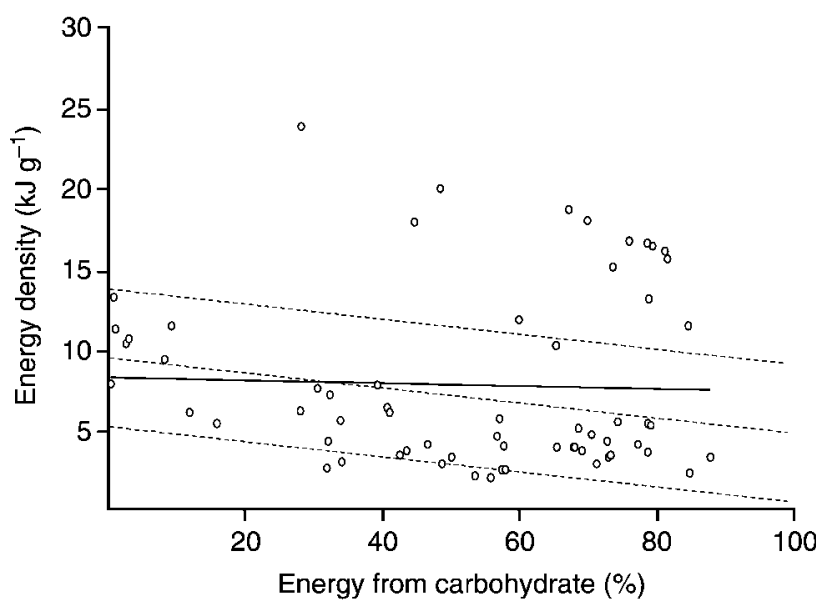

(c)

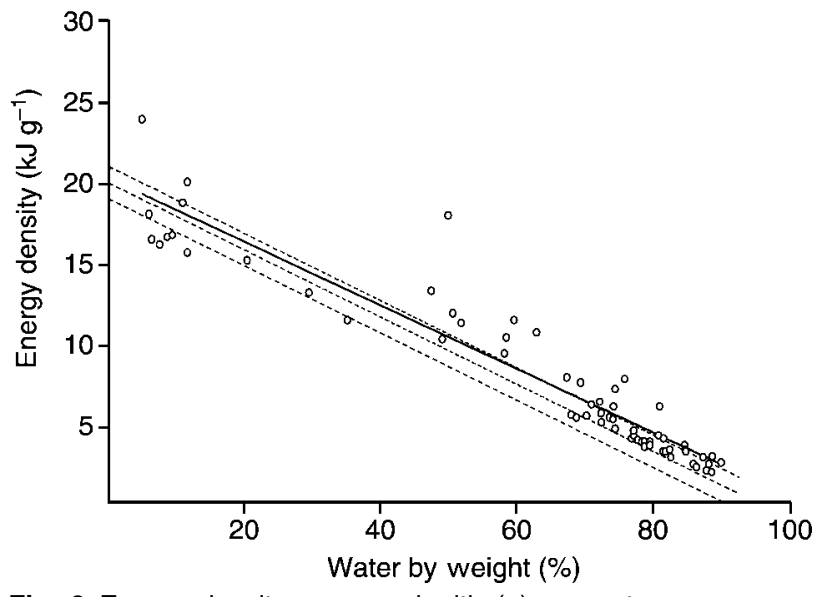

Fig. 2 Energy density compared with (a) percentage energy as fat, (b) percentage energy as carbohydrate and (c) percentage water by weight of reduced-fat foods. The three parallel dashed lines represent the Australian population diet (regression and 95\% prediction bands). Circles and solid regression line represent reduced-fat foods 
There appeared to be a cluster of RF foods that were low in fat $(<25 \%$ energy) but high in carbohydrate $(>60 \%$ energy) and $\mathrm{ED}\left(\sim 15 \mathrm{~kJ} \mathrm{~g}^{-1}\right)$, which clearly influenced the fat and carbohydrate relationships with ED. This group of products consisted of foods with low water content such as potato chips, biscuits, margarine, peanut butter and a chocolate bar.

There was a consistent negative relationship between water content and ED for RF foods $(\beta=-0.20,95 \% \mathrm{CI}$ -0.21 to -0.18$)$, FF foods $(\beta=-0.24,95 \%$ CI -0.26 to -0.22 ; data not shown) and the population diet ( $\beta=-0.208$, 95\% CI -0.209 to -0.207 ) (Fig. 2c). The clusters of RF, energy-dense products mentioned above can be seen to have low water content.

Figure 3 shows the relationships between ED and percentage energy as fat, percentage energy as carbohydrates and percentage water by weight for the $\mathrm{HF}$ vegetable-based dishes. The data points for the HF vegetable-based dishes have been superimposed on the relationships seen in the Australian diet.

There was no correlation between percentage energy as fat and $\operatorname{ED}(\beta=-0.02,95 \% \mathrm{CI}-0.06$ to 0.01$)$ (Fig. 3a) and a marginal positive correlation between ED and percentage energy as carbohydrate $(\beta=0.035$, 95\% CI 0.00-0.07) (Fig. 3b) for the HF vegetable-based dishes. The relationship between ED and percentage water by weight $(\beta=-0.17,95 \% \mathrm{CI}-0.19$ to -0.15$)$ is shown in Fig. 3c. There was an obvious clustering of the HF vegetable-based dishes at the high water content end of the relationship.

\section{Discussion}

This study assessed the relationships between energy density and dietary macronutrients within foods with reduced-fat claims, their full-fat equivalents and high-fat vegetable-based dishes. These relationships were contrasted with those found within the non-beverage content of the Australian diet.

While products with RF claims were significantly lower in fat and ED than their FF equivalents, they were higher in ED than the Australian diet. On the other hand, the HF vegetable-based dishes had more than $50 \%$ of their energy from fat, but their ED was less than that of the population diet. Differences in water content probably explained most of these differences in ED.

Overall, there was a proportional reduction in ED with a reduction in fat content from $\mathrm{FF}$ to $\mathrm{RF}$ equivalents. However, some food pairs showed much less change in ED than predicted, for example particular brands of potato chips, peanut butter and chocolate chip cookies. Other product pairs actually demonstrated a change in ED that was greater than expected, such as certain brands of yoghurt. There did not seem to be any distinguishing characteristics of products that were either higher or lower in ED than predicted. (a)



(b)

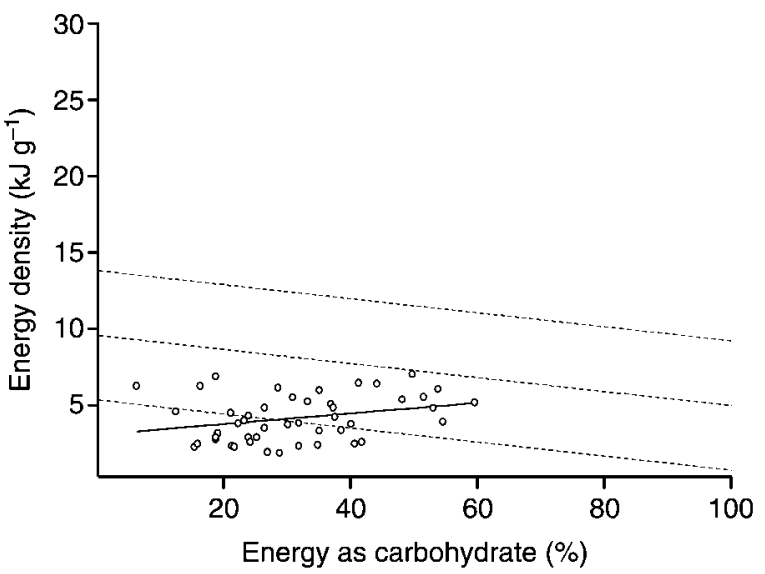

(c)

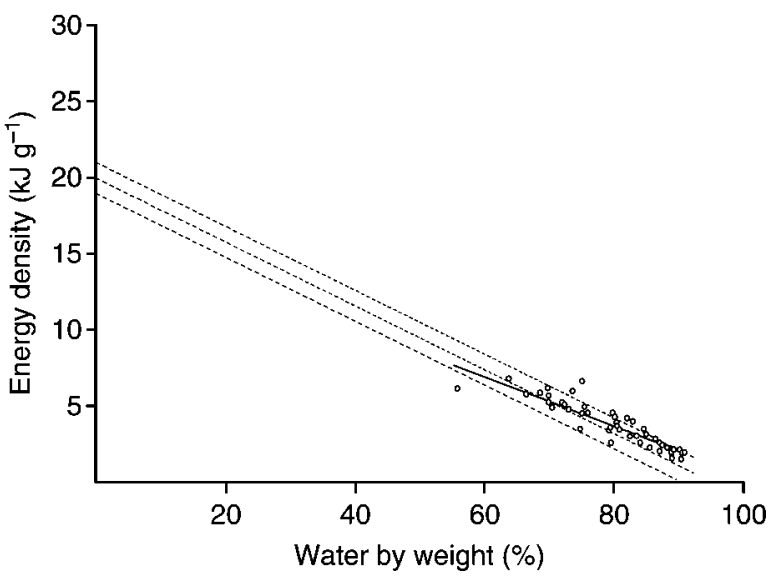

Fig. 3 Energy density compared with (a) percentage energy as fat, (b) percentage energy as carbohydrate and (c) percentage water by weight of high-fat, vegetable-based dishes. The three parallel dashed lines represent the Australian population diet (regression and 95\% prediction bands). Circles and solid regression line represent high-fat, vegetable-based dishes

Of particular interest were the relationships between ED and fat or carbohydrate within the Australian diet compared with the same relationships within the food groups we examined. The dietary intake data showed that ED was positively related to percentage energy as fat and 
negatively related to percentage energy as carbohydrate, and this has been shown to be the case for foods in general $^{17}$. However, we have identified two important food groups that appear to be exceptions to this general pattern: foods with RF claims and HF vegetable-based dishes. A cluster of energy-dense, RF products that were relatively high in sugar and low in water content seems to have been responsible for the loss of the positive relationship between $\mathrm{ED}$ and fat content in the RF product group. Within the HF vegetable-based dishes, the lack of a relationship between ED and fat content was unexpected. However, recipes with a very high percentage of energy as fat were dominated by more energy-dilute ingredients such as salad vegetables. The inclusion of ingredients such as rice, pasta and cous cous tended to increase the carbohydrate content and the ED simultaneously because it was 'replacing' the more energy-dilute base of vegetables.

It is important to emphasise that we have highlighted the exceptions to a general rule that still holds for most foods and diets: a high fat content means high ED. The evidence is compelling that an energy-dense diet promotes 'passive over-consumption' of total energy (see below) and weight gain, and that makes these exceptions important. A high intake of products with RF claims could increase the ED of the diet and promote weight gain. Alternatively, a high intake of vegetablebased dishes, even those with a lot of added oil, may decrease the ED of the diet and reduce the risk of weight gain.

Several studies $^{8-12}$ have examined the effect of changing dietary fat content without altering the ED of the overall diet (or meal). Although the amount of fat (between 20 and 60\% of energy) and other macronutrients consumed in each diet varied, the overall weight of food intake was relatively stable. Results indicate that subjects consumed similar ad libitum total energy intakes despite large variations in the composition of the diets. In addition, two studies ${ }^{18,19}$ changed the ED without altering the diet composition and again found that similar weights of food were consumed; however, because the ED of the diets varied, subjects consumed up to 1.7 times the amount of energy on the high-ED diet compared with the low-ED diet. Therefore, while HF diets are usually energy-dense and visa versa, it appears that it is the high ED, not the high fat content that drives passive over-consumption of total energy $^{20}$.

Why would people consume a large amount of RF products? Dietary recommendations and communications to the public from the nutrition community have long promoted the need for a reduction in fat intake ${ }^{2}$. Food manufacturers responded to the challenge to develop more RF options. In the USA, between 20 and $37 \%$ of new products have had nutrition claims for the 10 years leading up to 1997, with over half of those being for 'Low Fat' or

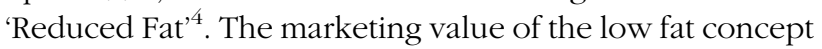

has been heavily exploited and, in many cases, overexploited. In Australia, the Code of Practice for nutrient claims in food labels and advertising are self-regulated and can be easily violated by deceptive marketing practices. Our study found several examples of this. Three products that made a 'Percent Fat Free' claim had the same macronutrient composition as an equivalent product sold by the same manufacturer with no claim made on the packaging. Three more products making a 'Lite' claim contained the same dry ingredients as the manufacturer's regular product, but were packaged in lighter packaging with instructions to use different additional ingredients for preparation. These marketing strategies are employed because consumers often give foods a more positive or negative image than they deserve based on the packaging and labelling ${ }^{21}$.

This heavy, and often misleading, marketing of RF products has led many consumers to perceive that these products are 'guilt-free', 3 and can be consumed without risk of weight gain. Several studies have shown that people will consume more of a product labelled 'Low Fat' because they feel it does not violate self-imposed dietary restrictions ${ }^{17,22,23}$.

A high consumption of products with RF claims may help to explain the so-called 'American paradox', where the increase in obesity prevalence has been parallelled by increases in the availability of 'Low Fat' products and reported increases in carbohydrate and total energy intakes ${ }^{24}$. Absolute intake of dietary fat appears to have remained stable ${ }^{3,25}$, although significant underreporting of fat intake is likely ${ }^{5}$. This over-concern with reducing fat intake may also prevent people from gaining the cardiovascular benefits from monounsaturated and polyunsaturated fats.

\section{Implications}

Nutrition messages about reducing dietary fat intake need to caution against over-consumption of products with RF claims. Food regulations in relation to RF claims need to be tightened to include energy density criteria and to ensure that the marketing of RF products does not imply that the products are 'guilt-free' or that they will promote weight loss. In addition, nutrition education messages about cardiovascular health can be more confident that the promotion of vegetable-based dishes that are relatively high in monounsaturated and polyunsaturated fats will not increase the risk of weight gain.

\section{Acknowledgements}

We thank Dr David Cameron-Smith and Professor Tony Worsley for their contributions to this paper. This study was undertaken by the Centre for Physical Activity and Nutrition Research and funded by Deakin University. 


\section{References}

1 Bray GA, Popkin BM. Dietary fat intake does affect obesity! American Journal of Clinical Nutrition 1998; 68(6): 1157-73.

2 Astrup A, Grunwald GK, Melanson EL, Saris WH, Hill JO. The role of low-fat diets in body weight control: a meta-analysis of ad libitum dietary intervention studies. International Journal of Obesity and Related Metabolic Disorders 2000; 24(12): $1545-52$.

3 Sigman-Grant M. Can you have your low-fat cake and eat it too? The role of fat-modified products. Journal of the American Dietetic Association 1997; 97(Suppl. 7): S76-81.

4 Weimer J. Accelerating the trend toward healthy eating. In: America's Eating Habits: Changes and Consequences Washington, DC: US Department of Agriculture, Economic Research Service, 1999; 385-401.

5 Astrup A. The American paradox: the role of energy-dense fat-reduced food in the increasing prevalence of obesity. Current Opinion in Clinical Nutrition and Metabolic Care 1998; 1(6): 573-7.

6 Heini AF, Weinsier RL. Divergent trends in obesity and fat intake patterns: the American paradox. American Journal of Medicine 1997; 102(3): 259-64.

7 National Health and Medical Research Council. Acting on Australia's Weight. Canberra: National Health and Medical Research Council, 1997.

8 Stubbs RJ, Harbron CG. Covert manipulation of the ratio of medium- to long-chain triglycerides in isoenergetically dense diets: effect on food intake in ad libitum feeding men. International Journal of Obesity and Related Metabolic Disorders 1996; 20(5): 435-44.

9 Rolls BJ, Bell EA, Castellanos VH, Chow M, Pelkman CL, Thorwart ML. Energy density but not fat content of foods affected energy intake in lean and obese women. American Journal of Clinical Nutrition 1999; 69(5): 863-71.

10 Bell EA, Rolls BJ. Energy density of foods affects energy intake across multiple levels of fat content in lean and obese women. American Journal of Clinical Nutrition 2001; 73(6): 1010-8.

11 Bell EA, Castellanos VH, Pelkman CL, Thorwart ML, Rolls BJ. Energy density of foods affects energy intake in normalweight women. American Journal of Clinical Nutrition 1998; 67(3): 412-20.

12 Saltzman E, Dallal GE, Roberts SB. Effect of high-fat and lowfat diets on voluntary energy intake and substrate oxidation: studies in identical twins consuming diets matched for energy density, fibre, and palatability. American Journal of Clinical Nutrition 1997; 66(6): 1332-9.
13 Blundell JE, King NA. Over-consumption as a cause of weight gain: behavioural-physiological interactions in the control of food intake (appetite). Ciba Foundation Symposium 1996; 201: 138-54, discussion 154-8, 188-93.

14 Food Standards Australia New Zealand. Australian Food and Nutrient Database [online], 1999. Available at http://www.foodstandards.gov.au/recallssurveillance/ foodcompositionprogram/ausnut1999/ ausnutguideexplanatorynotes/index.cfm.

15 National Health and Medical Research Council. Dietary Guidelines for Australians. Canberra: National Health and Medical Research Council, 1992; 21-7.

16 DiMeglio DP, Mattes RD. Liquid versus solid carbohydrate: effects on food intake and body weight. International Journal of Obesity and Related Metabolic Disorders 2000; 24(6): 794-800.

17 Yao M, Roberts SB. Dietary energy density and weight regulation. Nutrition Reviews 2001; 59(8 Pt 1): 247-58.

18 Stubbs RJ, Johnstone AM, O'Reilly LM, Barton K, Reid C. The effect of covertly manipulating the energy density of mixed diets on ad libitum food intake in 'pseudo free-living' humans. International Journal of Obesity and Related Metabolic Disorders 1998; 22(10): 980-7.

19 Stubbs RJ, Johnstone AM, Harbron CG, Reid C. Covert manipulation of energy density of high carbohydrate diets in 'pseudo free-living' humans. International Journal of Obesity and Related Metabolic Disorders 1998; 22(9): 885-92.

20 Poppitt SD. Energy density of diets and obesity. International Journal of Obesity and Related Metabolic Disorders 1995; 19(Suppl. 5): S20-6.

21 Oakes ME, Slotterback CS. What's in a name? A comparison of men's and women's judgements about food names and their nutrient contents. Appetite 2001; 36(1): 29-40.

22 Miller DL, Castellanos VH, Shide DJ, Peters JC, Rolls BJ. Effect of fat-free potato chips with and without nutrition labels on fat and energy intakes. American Journal of Clinical Nutrition 1998; 68(2): 282-90.

23 Shide DJ, Rolls BJ. Information about the fat content of preloads influences energy intake in healthy women. Journal of the American Dietetic Association 1995; 95(9): 993-8.

24 Stubbs J, Ferres S, Horgan G. Energy density of foods: effects on energy intake. Critical Reviews in Food Science and Nutrition 2000; 40(6): 481-515.

25 Allred JB. Too much of a good thing? An overemphasis on eating low-fat foods may be contributing to the alarming increase in overweight among US adults. Journal of the American Dietetic Association 1995; 95(4): 417-8. 